\title{
A Fusion of the School Arithmetic and the School Algebra
}

\author{
Milosav M. Marjanović \\ Serbian Academy of Sciences and Arts, Belgrade, Serbia \\ Email: milomar@beotel.net
}

How to cite this paper: Marjanović, M.M. (2018) A Fusion of the School Arithmetic and the School Algebra. Open Access Library Journal, 5: e4889.

https://doi.org/10.4236/oalib.1104889

Received: September 6, 2018

Accepted: September 25, 2018

Published: September 28, 2018

Copyright $\odot 2018$ by author and Open Access Library Inc.

This work is licensed under the Creative Commons Attribution International License (CC BY 4.0).

http://creativecommons.org/licenses/by/4.0/

\begin{abstract}
In this paper we present our views on algebra as a school subject. To say precisely what is the content of this subject, the language of abstract algebra has to be used. Accordingly, this content consists of the establishing and application of the properties of operations in the system of natural numbers which are then carried over to the extended systems. In the last of these extensions-the set of rational numbers, these properties can be taken to be axioms of ordered field and the main facts that are deduced from them. Of course, in the classroom practice, this content is didactically transformed and shaped to serve the learner and is aimed at the development of the skill of transforming numerical and literal expressions. On the other hand, the school arithmetic, when structured properly, consists of the gradual building of the number blocks up to 10 , to 20 , to 100 and to 1000 , each of them having its own package of didactical tasks that are supposed to be accomplished. We take that both school arithmetic and school algebra have for their intuitive ground, the phenomenology which consists of sets at the sensory level and their configurations in the form of additive and multiplicative schemes. And while arithmetic deals with specific numbers denoted by their decimal notations, algebra deals with species of numbers (variables) denoted by letters. As for the properties of operations, in arithmetic they are related to specific schemes whose extent is determined by specific numbers, while in algebra they are related to the species of such schemes where letters replace specific numbers. The way we view school arithmetic and school algebra inspires us to suggest a fusion of these two subjects. In this paper we sketch how this fusion should be carried out, avoiding any possible abrupt semantic jumps from specific to general cases. The ultimate aim of teaching and learning both, arithmetic and algebra is the building of the systems of natural numbers, integers and rational numbers and as this aim is attained in the last classes of elementary school, in this paper we confine our considerations to the primary level. At this level we suggest and sketch: Elaboration of arithmetic in the form of gradual building
\end{abstract}


of number blocks, based on the permanent meaning of addition and multiplication. Derivation of properties of operations and their procedural expression from the very beginning (within blocks of numbers up to 20 and 100). Teaching situations which are selected to help and encourage development of the idea of variable (letters in the role of the unknown, evaluation of literal expressions, etc.). Derivation of properties of operations in symbolic form, based directly upon the experience of species of schemes.

\title{
Subject Areas
}

Education

\section{Keywords}

Additive and Multiplicative Schemes, Procedural and Symbolic Expression, Fostering the Idea of Variable, Derivation of the Properties of Operations

\section{Introduction}

\author{
Toute science est létude d' une \\ phenomenology ... toute pheno- \\ ménologie doit être regardée com- \\ me un "spectacle" visual.
}

René Thom [1]

Grecian logistica numerosa was a school subject designed for children to teach them calculate and that art was also considered to be indispensable for everyday life. Exactly the same may be said for school arithmetic today. What has been changed progressively are the methods of its elaboration. At the times of great innovators J. A. Comensky, J. H. Pestalozzi and their followers, learning process was directed so as to start with sensory experience and to lead to the creation of clear concepts. There exists a large variety of contemporary approaches to the elaboration of school arithmetic. A common characteristic of them all is the tendency to have the teaching matter well illustrated by an ample use of colorful pictures. We do not find to have a ground upon which one of these approaches would take to be exemplary. Thus, one aim of this paper is the sketching of an approach of elaboration of arithmetic structured in the form of number blocks up to 10 , to 20 , to 100 , to 1000 etc., each having a package of didactical tasks attached that have to be accomplished. Without any other pretension, this approach is also the one that this author has studied the most.

Discovery of Vieta's logistica speciosa had a great impact on the development of mathematics. The use of letters to denote species of numbers led to the idea of variable and to creation of literal algebra which, in the first half of $19^{\text {th }}$ century, was founded on its own axioms (Peacock, De Morgan et al.).

Becoming a school subject (mostly during the first half of $19^{\text {th }}$ century), 
teaching and learning algebra caused a lot of problems and this subject was considered to be based upon "the rules without reason". The old fashioned arithmetic reduced to the art of denoting numbers in the positional system and the performance of four arithmetic operations on these notations had the emphasis on carrying lengthy calculations with mechanical speed and accuracy. Of course, a natural link between such arithmetic and algebra could not exist, what caused algebra to be cut off from its meaning. The search for reasons of rules of school algebra, started in the latte decades of the $20^{\text {th }}$ century, has opened a large avenue of research. The books [2], [3] and [4] provide a quite complete presentation of the state of affairs in this area of research.

Rules of school algebra are the properties of operating with literal expressions which are composed of letters denoting species of numbers (variables) combined with operational signs. Literal expressions get a meaning when linked with those in arithmetic which are composed of notations for specific numbers also combined with operational signs. The meaning of arithmetic expressions is related to various patterns of objects (which are usually dots, circlets, etc. as often seen in the school books). When the structure of these patterns is not fixed but their representation suggests their variability (as, for example, it is a rectangular arrangement having $k$ rows and $l$ columns), then such species of patterns are an intuitive basis for the meaning of algebraic expressions. Historically, algebra was considered to be the "the doctrine of solving equations", while the school algebra is primarily an art of dealing with literal expressions. We consider both, school arithmetic and school algebra to have for their ultimate aim construction of the systems of natural numbers, integers and rational numbers.

To say it formally and precisely, school algebra is the way how the key idea of variable is acquired and exploited and how the properties of operations are established and applied in the system of natural numbers and then carried over to the extended systems. In the last of these extensions-the set of rational numbers, these properties can be taken to be axioms of the ordered field and the facts deduced from them. Here we use the language of abstract algebra, but in the classroom practice all these facts are (or, better to say, should be) didactically transformed and shaped so as to serve best the learner. The second aim of this paper is a sketch of the way how the first ideas of algebra are elaborated in the primary school, bearing in mind their link with the arithmetic contents.

We continue with making some general observations about the way how concepts are formed at this early stage of learning.

Learning arithmetic, the concepts are created gradually in such a way that children experience a long series of corresponding examples that are embedded in everyday situations. Some accompanying questions and requirements direct attention and activities of children in whose mind an inner representation is formed, called mental image and who assimilate a word to serve as the name of the concept. From such activities a concept results as a tripartite entity consisted of corresponding examples, mental image and name. In psychology, all unessen- 
tial properties of examples corresponded to a concept (i.e. those properties that some of these examples have and some others do not) are collectively called noise. It is usually easier to say which properties are unessential and their ignoring (neglecting) is often a more realistic way of abstracting. In the most cases, creation of concepts consists of selecting their corresponding examples and of deliberate neglecting of their unessential properties, what leaves behind their characteristic properties.

The inscribed motto indicates a phenomenology as the realm of meaning of a science. In the case of arithmetic the underlying phenomenology consists of groups of visible objects in the surrounding space and of manipulation with them. Pictures and drawings that represent such groups of objects, when they are not immediately perceived, are also included and called pictograms, as well as those drawings which are iconic signs of concepts. This latter kind of pictorial signs are the examples of concepts having as little noise as possible. Exactly in that sense they have to be taken as represents of concepts, usually called ideograms and it would be a wrong idea to identify them with abstract entities as the concepts are. Typical examples of ideograms are geometric drawings and, in arithmetic, number pictures which are regular patterns of objects (dots, circlets, etc.), number of which is projected at the first glance. Let us add that at the early stages of learning mathematics the iconic representations are important conveyors of meaning.

As the concept of set is taken to be more general than all other concepts of classical mathematics, its examples exist at all levels of abstraction. The lowest of these levels is the case when the examples are groups of real world objects in the surrounding space. At somewhat higher level the examples of sets whose elements are conventional symbols (letters, decimal notations for natural numbers, etc.) are used, continuing then up to the levels when the elements of sets are also abstract concepts (the set of natural numbers, the set of real numbers, etc.). For the groups of visible objects in the surrounding space the term sets at the sensory level is used. These sets and manipulation with them represent the underlying phenomenology upon which arithmetic is based. Hence we should not overlook the dependence of conception of natural numbers on the experiencing sets at sensory level and the manipulations with them.

As we know it from the history of mathematics, when the Pythagoreans discovered incommensurable quantities, it threw into question measuring in geometry. And it was Eudoxus who overcame that crisis, extending the earlier (Pythagorean) idea of number as the ratio of two natural numbers to the ratios of two quantities of the same kind, creating so the theory of (positive) real numbers. Since arithmetic in Antiquity was rhetoric, numbers represented by infinitely many equivalent ratios of quantities and the operations performed on quantities (not on numbers), this all made this great creation technically very complex and tedious. Almost two millennia later, Descartes discovered the number axis as the model for real numbers, where each number is uniquely represented by an in- 
terval having an end point at the origin $O$ and where this model is closed for constructions on these intervals (i.e. for operations on numbers). This significant simplification of Eudoxus' theory permitted the number axis to enter school books and to be an inevitable didactical device for learning real numbers.

Natural numbers are embedded into the system of real numbers and, on the number axis, they are represented by intervals which are unions of sequences of adjunctive unit intervals. The right ends of these intervals form a monotonous sequence of marks which is conceived as being unbounded, what is an intuitive ground for conception of the infinite set of natural numbers. Graphical representation of the number axis is only an iconic sign which stands to suggest its whole structure. Identifying this structure with the seen sign is a naïve mistake and the cause for perplexities. Marks on various scales used for measuring are examples of sets at the sensory level as it is also the drawing representing the number axis when taken without the meaning it suggests (A drawing, often seen in primary school books, of a sequence of marks enumerated with numbers up to 20 and with a jumping frog does not seem to be a very clever device to represent addition and subtraction in this block of numbers).

\section{Learning Arithmetic-A Brief Survey of the Main Teaching Themes}

The knowledge of natural numbers that is gained through learning in school is essential and it deserves a brief recapitulation in terms of didactics of mathematics. Such an account is of interest not only for primary teachers but also for all mathematics teachers and we will present it here in the form of a series of the main teaching themes. This presentation will conjure up to some degree everybody's experience from the years spent in school.

\subsection{Counting up to 10-An Introductory Theme of Teaching Arithmetic}

Reciting the number names in correct order up to 10 is the only case of genuine counting, which children usually learn at their parents' home and then practice systematically in the classroom. At this stage children do not learn to write numbers, their attention is directed to (illustrated) situations, understanding of which makes sense to the questions as "How many altogether", "How many left", "How many more (less)", etc. that are answered orally. Groups of objects to be counted are small (not exceeding 10 in number) and the main didactical task of this theme is preparation of children to understand fully the meaning of usual questions with which the teaching of arithmetic starts.

\subsection{Cantor Principle of Invariance of Number}

Perception of sets precedes the conception of numbers. Cantor describes this cognitive process that leads to the idea of cardinal number in its natural dependence on sets of observable objects as the result of two abstractions: ignoring of 
all properties except ordering and then ignoring the ordering itself (see [5]). Modifying slightly this formulation to make it more suitable for didactical purposes, we express Cantor principle of invariance of number in the following way:

Starting with the observation of a set of visible objects and abstracting (forgetting)

1) the nature of these objects, and

2) any kind of their organization, the abstract idea of number results.

When we say organization we mean any way how the elements of a set can be ordered, arranged, grouped etc. Following Cantor, when a set is denoted by $S$, the number of its elements is denoted by $\overline{\bar{S}}$ where two overbars indicate two above abstractions. Let us also say that this cognitive principle stands for the formal concept of equivalent sets expressed in terms of one to one correspondences.

We see the role of this principle at the very beginning when the elements of various sets are counted and when they are attached to the number names in different order. Particularly important is the case when different expressions corresponding to the different groupings of elements of a set are equated since they represent one and the same number.

In proceeding further let us note that Vigotskii [6] calls a class of mutually related concepts a system of concepts. In mathematics such a class is called a (concrete) mathematical structure. In psychology, reflection in mind of such a class is called a mental scheme. In what follows we will be concerned with building of number blocks up to 10 , up to 20 , up to 100 and up to 1000 which consist of concepts of individual natural numbers and operations with them. These blocks will be specific examples of systems of concepts.

\subsection{Block of Numbers up to 10}

We summarize here what is done when this block is built and which didactical tasks are accomplished.

The meaning of numbers $1,2, \ldots, 10$ and 0 is established by relating each of them with the corresponding sets of various objects.

Each of these numbers is treated as an individual concept denoted symbolically by its digit and associated with its number image, being an arrangement of uniform signs, number of which is projected at the first glance.

A somewhat more delicate is the case of zero which is related to the materialization of the empty set as being the "empty place". For example in a sequence of boxes containing less and less marbles the last empty one is an example of the empty place.

Each digit is written in a number of moves of the hand that are made in a fixed order. Exercising, these moves tend to be more and more regular and the handwriting becomes nicer. It is important that children learn to write digits correctly from the very beginning.

Children are given the examples of situations which can be modeled as the 
union of two disjoint sets. Such an idea is called an additive scheme and when the numbers of elements of these sets are given and the number of elements of their union is to be found, it is said that this scheme is followed by an addition task. As soon as the children are aware of an additive scheme followed by an addition task, they write a sum to denote the sought number. This meaning of addition is permanent and should not be confused with a more technical one, when sums are calculated to find the unique decimal notation for their value.

When the additive scheme is imagined or experienced (pictorially represented) and when the numbers of elements of the union and of the one of these sets are given and the number of elements of the other set is to be found, then we say that this scheme is followed by a subtraction task. Examples of situations of this kind provide a permanent meaning to subtraction.

Let us note that, in the classroom practice, additive schemes are mostly given in the form of simple pictures-the sets are "small" (their number of elements does not exceed 10) and the sought numbers are found by counting (But this is not the reason for the teachers not to recognize the importance of the activities when the meaning of these two operations starts to be formed).

Children write two different sums when they denote the number of elements of the union of two disjoint sets taken in two different orders. By equating these sums they discover the rule of the interchange of summands. Teachers should understand that this rule is one of the principles of arithmetic which is intuitively acceptable but not to be verified by counting (or calculation).

A sum (a difference) denotes a number which is called its value. To calculate a sum (a difference) means to express its value by its unique decimal notation. By doing a sufficient number of exercises, children are trained to calculate quickly sums and differences when their value is within this block. As already said, "calculation" at this stage reduces to counting and is based on the imagery that children develop in contact with the number images.

Numbers up to 10 , with the operations of addition and subtraction and the relation "to be larger than" constitute a system of concepts (a mathematical structure) that we denote by $\left\{N_{10},+,-,<\right\}$.

\subsection{Block of Numbers up to 20}

Majority of children learn spontaneously to count up to 20, reciting the number names in order. But counting is not taken to be a basis upon which larger number blocks are built. A sum as, for example, $10+7$ already has its meaning established in the block of numbers up to 10. It denotes the number of elements of the union of two sets, one having 10 elements and the other one 7. Decimal notation for that number is not included in the material of which block of numbers up to 10 consists. But the use of such notations is the way how this block is extended to the block of numbers up to 20 .

Namely, the sums $10+1,10+2, \ldots, 10+10$ are denoted briefly as $11,12, \ldots$, 20 respectively and children learn their names eleven, twelve, ..., twenty. By 
equating two notations for the same number, the equalities $10+1=11,10+2=$ $12, \ldots, 10+10=20$ are established.

The following properties of addition and subtraction are established: the rule of association of summands, the rule of interdependence of addition and subtraction, the rule of subtracting a number from a sum, the rule of subtracting $\alpha$ sum from a number, the rule of interchange of the subtrahend and the difference, etc. Application of these rules is not a very simple task for children. Thus, the exercises of this kind have to be programed using the technique of place holders.

Methods of adding and subtracting, when the 10-line is crossed are the first genuine cases of calculation and these methods are worked out in detail. By practicing these methods, children learn to calculate quickly the results of all entries of addition table. Therefore, the block of numbers up to 20 is a natural framework within which the addition (and subtraction) table (tables) is (are) formed.

\subsection{Place Holders-An Acted-Out Arithmetic}

A variety of graphical signs as number boxes, circlets, shorter and longer underlines, etc. are often used to execute some specific tasks in programed learning. For example, in arithmetic textbooks these signs are seen applied in the cases as the following are

$$
2+4=\ldots, \quad 5+\ldots=14, \quad 7 \times \ldots=63, \text { etc. }
$$

Previous generations of teachers used to express such assignments in words: "Find the sum $2+4$ ", "Which number must be added to 5 to make 14 ", "How many 7's you must take to make 63", etc. When these assignments are expressed orally, a pupil has to remember the verbal information first, prior to the giving an answer. Set down in the programed form, such assignments keep the whole information which can be automatically read off, even without converting it into a sentence. Namely, as the school practice shows it, children learn easily what is required to be done, filling in the blank spaces indicated by these graphical signs. Called place holders (or space holders) these signs have an evident pedagogical justification being usable as an invaluable tool in elaboration of arithmetic.

Let us select here a couple of examples related to the methods of adding up over ten and subtracting down below ten. For the sake of understanding, in the beginning, children are supposed to accomplish all intermediate steps. The following examples illustrate it.

$$
7+8=7+3+\ldots=
$$$$
16-9=16-6-
$$
$-3=$

To respond correctly, children fill in the blank places, writing the numbers which preserve these equalities. In these examples such numbers are: 5, 10, 15 in the former case and 3, 10, 7 in the latter. As it is seen, in different places different numbers are written. Thus, the place holder technique is used to hold blank places and it is a wrong idea to assign them any numerical value. The inevitable use of this technique is needed for active learning of arithmetic, fostering so both 
the understanding and the automatic performance of routine tasks.

\subsection{Block of Numbers up to 100}

The sums $20+1,20+2, \ldots, 20+10$ and their decimal notations $21,22, \ldots, 30$ make the first step in extension. Then $30+1,30+2, \ldots, 30+10$ and their decimal notations $31,32, \ldots, 40, \ldots, 90+1,90+2, \ldots, 90+10$ and their decimal notation $91,92, \ldots, 100$ are the numbers of this block. The reader will notice that addition, not the counting, is the basis of this extension.

The technique of vertical addition (including the cases when one ten is carried over) and vertical subtraction (including the cases when one ten is borrowed) are presented in detail. Number images are employed to illustrate these procedures and to supply them with meaning.

These procedures are also described in terms which underline the decimal stricture of the manipulated numbers but as soon as these procedures become more automatic, such descriptions are suppressed obtaining abbreviated form of the inner speech (For the meaning of inner speech, see [6]).

Up to this stage the block of numbers up to 100 is an additive structure which we denote writing $\left\{N_{100},+,-,<\right\}$. But this block is also a natural range of numbers within which the meaning of multiplication and division is established.

A situation which can be modeled as a finite family of disjoint finite equipotent sets is called a multiplicative scheme. When the number $m$ of the members of this family and the number $n$ of the elements of these sets are given and the number $p$ of the elements of their union is to be found, we say that this is a multiplication task that follows this scheme. When $p$ and $n$ are given and $m$ is to be found, we say that this is a division task, called partition, which follows this scheme and when $p$ and $m$ are given and $n$ is to be found, we also say that this is a division task, called quotation, which follows this scheme.

Equivalent ways of representing a multiplicative scheme as a rectangular arrangement or, in the set theory, a direct product can also be an alternative.

When building the multiplication table all included products are related with their iconic representations which suggest a quick calculation of their value. Following illustrations, children are supposed to take an active part in the building of this table as well as these representations serve children to memorize easier the values of these products.

Properties of multiplication are established and expressed: the rule of interchange of factors, the rule of association of factors, the rule of multiplication of sums and differences by a number, etc. A collection of $m$ boxes each containing $n$ marbles is a suitable model of the multiplicative scheme. Similarly, $k$ packages, each containing $m$ boxes and each box containing $n$ marbles is a suitable model for the triple product. Teachers should be aware of the role of the rule of association of factors (the associative law for multiplication) which reduces triple and multiple products to the products of two numbers. Thus, it is enough to form the multiplication table in the case of products of two numbers as well as to ex- 
press properties of multiplication in terms of products of two numbers.

The following properties of division are established and formulated: the rule of interdependence of multiplication and division, the rule of interchange of the divisor and the quotient, the rule of division a sum and a difference by a number, etc.

By doing exercises, children learn the procedure of division with remainders and particularly essential are so called canonical divisions: by 2 up to 19, by 3 up to $29, \ldots$, by 9 up to 89 . These cases of division are supposed to be done mentally and, together with the addition and multiplication tables, they enter long term memory store.

With multiplication and division, the block of numbers up to 100 becomes a still richer structure which is denoted by $\left\{N_{100},+,-, \times_{.}:,<\right\}$.

\subsection{Block of Numbers up to $\mathbf{1 0 0 0}$}

Products: $2 \times 100,3 \times 100, \ldots, 10 \times 100$ have their meaning already established in the block $N_{100}$. Denoting them by their decimal notations $200,300, \ldots, 1000$ the hundreds of the first thousand are introduced. Then the sums of these hundreds and two digit numbers as, for example, $200+18,300+71,900+54$, etc. also obtain their decimal notations $218,371,954$ etc. what gives meaning to the numbers up to 1000 .

Vertical addition and subtraction of two three digit numbers is worked out in detail. Formal operations on digits are illustrated by means of number images and such illustrations provide a full understanding of these procedures. Description of these activities, intentionally leaving out details, also turns into the forms of the inner speech.

Multiplication and division of three-digit numbers by one-digit numbers is also illustrated by the use of number images and a thorough elaboration of this theme is a necessary introduction to the more complex cases of multiplication and division by multi-digit numbers.

Our analysis demonstrates it clearly that the explanation of the steps made in performing addition and subtractions based on rules of arithmetic is rather awkward. Instead of it, vertical addition and subtraction, when columns of units, tens and hundreds are formed evidently has an advantage. This remark equally holds in the case of operations with multi-digit numbers.

At the end, let us remark that the blocks of numbers up to 20 and up to 100 are the stages of learning arithmetic when the permanent meaning of arithmetic operations is established and where the rules expressing the main properties of these operations are derived (In classroom practice, pedagogical reasons may postpone, of course, derivation of some of these rules to the classes that follow). In that respect, these stages are a particularly subtle period of learning arithmetic. The block of numbers up to 1000 is just a prelude to the technical tasks of learning algorithms of operating with the multi-digit numbers. Without any intention to diminish the importance of these algorithms, here we put emphasis on 
meaning and properties of arithmetic operations.

\section{Beginnings of School Algebra. Fostering the Idea of Variable}

In the period of rhetoric algebra, a specific example of a type of equations was done to demonstrate that analogously all other cases have to be done. Similarly, when teaching arithmetic, the properties of operations (rules of arithmetic) are expressed in words (rhetorically) and then applied to specific cases. Now we list the prerequisites for derivation of these rules.

Children are used to write sums and differences whenever they recognize additive schemes, followed by addition and subtraction tasks, respectively as well as they are used to write products and quotients whenever they recognize multiplicative schemes followed by multiplication and division tasks, respectively.

Let us call sums, differences products and quotients basic expressions. Children know that a component of a basic expression which is a sum or a difference is written with brackets put round it. Brackets are understood as a command: first do what is written in brackets.

Children know to calculate the value of simple expressions, when such exercises are programed with place holders acting as the silent hints.

When a scheme is seen in two different ways, two different expressions are written. Denoting one and the same number (i.e. having the same value) such two expressions are equated. Different way of grouping elements of a set leads to different expressing of the number of its elements what, according to the Cantor principle, does not effects its cardinality. This procedure of equating expressions is the ground upon which rules of arithmetic are derived.

Let us remark that the term "procedural knowledge" is taken with a negative connotation meaning that a procedure is carried out without a necessary understanding. But when a piece of knowledge is assimilated with full understanding and expressed example by example, then this manner of expressing we will call procedural (without packing any negative connotation). For example, the ways how the algorithms of performing four arithmetic operations on multi-valued numbers are procedural-having demonstrated them in a number of specific cases, then it is expected that all other cases will be done analogously.

\subsection{Derivation of Arithmetical Rules and Procedural Way of Their Expression}

Already within the block of numbers up to 20 , the rule of interchange of summands, the rule of association of summands and the rule of interdependence of addition and subtraction are derived and expressed procedurally. At this level of teaching disjoint sets and their families are represented by some more concrete models, say, by boxes of marbles and their packages.

In order to illustrate this way of derivation of rules of arithmetic, let us consider the case of derivation of the rule of multiplication a sum by a number (dis- 
tributive law) which is attached to the block of numbers up to 100 .

(Imagine that a teacher leads and his/her class follows him/her with active participation).

There are 6 packages each containing a red box with 7 marbles in it and a blue box with 5 marbles in it. Altogether, there are $7+5$ marbles in each package. In 6 packages there are $6 \times(7+5)$ marbles. In 6 red boxes there are $6 \times 7$ marbles and in 6 blue boxes there are $6 \times 5$ marbles. Altogether, there are $6 \times 7+6 \times 5$ marbles. Equating two expressions which denote the total number of marbles in the packages, we write

$$
6 \times(7+5)=6 \times 7+6 \times 5
$$

(An illustration of 6 packages of boxes with numbers inscribed on their lids would surely be helpful to children in following this long text). This example is then varied changing the data and including children in the activity. For example, the teacher asks: what would you write if there were: a) 3 packages, b) 5 packages, c) 7 packages?

$$
\begin{aligned}
& \text { a) } \_\times(7+5)=\ldots \times 7+\ldots \times 5, \\
& \text { b) } \_\times(7+5)=\ldots \times 7+\ldots \times 5, \\
& \text { c) } \_\times(7+5)=\ldots \times 7+\ldots \times 5 .
\end{aligned}
$$

What would you write if there were: a) 3 marbles in the red box, b) 5 marbles in the red box, c) 6 marbles in the red box?

$$
\begin{aligned}
& \text { a) } 6 \times(\ldots+5)=6 \times \ldots+6 \times 5 \text {, } \\
& \text { b) } 6 \times(\ldots+5)=6 \times \ldots+6 \times 5 \text {, } \\
& \text { c) } 6 \times(\ldots+5)=6 \times \ldots+6 \times 5 \text {. }
\end{aligned}
$$

Then, such variations should follow changing the number of marbles in the blue box, etc. and, at the end, varying simultaneously all three numbers of packages and of marbles in both boxes.

(Here we see how inevitable is the technique of place holders without which the explanation of what has to be done would be just inadmissible rigmarole of words.)

All relations that express rules of arithmetic continue to hold true when the involved numbers are replaced by any other numbers. For example, when in the relation $6 \times(7+5)=6 \times 7+6 \times 5$ the numbers $6,7,5$ are replaced by any other three numbers, the relation so obtained continues to hold true. For a relation having this property we say that it has invariant form. In the following six examples:
a) $3+4=5+2,3+4=(3+2)+(4-2)$,
b) $(3+4)+7=10+4,(3+4)+7=3+(4+7)$
c) $7 \times(10-1)=7 \times 10-7,7 \times(10-1)=7 \times 10-7 \times 1$

all relations are true but, in all examples under a), b) and c), only the second of them has invariant form.

(When subtraction or division figures in a relation then the involved numbers should be replaced by the numbers for which these operations are feasible.)

When a rule has been derived and elaborated properly, its symbolic form is 
obtained replacing the involved numbers by letters. Children easily accept this procedure of replacing if they are already experienced to use letters in the role of variable. Now we focus our attention to those teaching and learning situations which are intended to serve developing of the idea of variable.

\subsection{Steps Taken to Develop the Idea of Variable}

Historically, the first manifestation of the idea of variable was the use of letters to denote the unknowns. It is exactly the same when mathematics is taught and learnt, the first step is the using of letters to denote unknown numbers. The jottings as, for example, $x=5$ or $x+5$ have no a priori meaning. Therefore some activities have to be planed through which such an equality and such an expressions gain the meaning.

The way how children are told that a letter, say $X$, is used to denote an unknown number is, for example, the following:

The teacher shows to his/her class picture (or a model) of a box with $x$ written on its lid. "When the box is closed, can we know how many marbles there are inside it?", the teacher asks "No, we can't", the children would answer in one voice. "We write $x$ to denote that unknown number", says the teacher. Then he/she shows another picture of that box with its lid lifted up (or lifts up the lid). The marbles are counted and it is found that there are them 8 . "Now the number of marbles is known and we write $x=8$ ", the teacher continues. Repeating such and similar exercises, the children acquire the meaning of $x$ when it denotes an unknown number as well as the meaning of the equality $x=8$, etc.

A model for the additive scheme can be two boxes (or their picture) with marbles in them. To such representation of this scheme, exercises of attaching them sums and differences should be done. When one of three numbers: $k$-the number of marbles in the first box, $l$-the number of marbles in the second box, $s$-the total number of marbles is unknown, the equations $x+I=s, k+x=s, s-$ $x=k, s-x=l$ arise. They are solved applying the rule of interdependence of addition and subtraction and the rule of interchange places of subtrahend and difference. For example, the equation $s-x=k$ is solved writing $x=s-k$.

A quite convenient model for a multiplicative scheme is a rectangular arrangement having $k$ rows and $l$ columns. Usually dots or circlets are used to form such arrangements. Let $p$ be total number of dots, then when one of the numbers $k, l, p$ is unknown, the equations $k \times x=p, x \times l=p, p: x=k, p: x=1$ arise. They are solved applying the rule of interdependence of multiplication and division and the rule of interchange of places of divisor and quotient. For example, the equation $p: X=l$ is solved writing $\mathrm{x}=p: I$ (Here we use letters to denote the types of these equations. In the classroom practice, instead of letters, specific numbers from the block which has been already elaborated are used).

Solving these equations, one by one, the value of $x$ varies running through a set of numbers. Thus $x$ in the role of an unknown number is an example of variable and, probably, the easiest such case to be approached. Let us remark that it 
suffices to confine the equation solving to these simple, basic types, when the task of developing the idea of variable is concerned (A tendency to solve more complex types of equations in primary classes seems to not be well founded because, at this level-neither children acquire with full understanding the idea of equivalent conditions nor there is any good reason for such premature formalism).

Exercises when given numbers are tried to see which of them is the solution of a given equation "forces" $x$ to run through a set of possible values. Such exercises also strengthen the role of the unknown as a variable.

Another type of instructive exercises, having this same purpose, is the case when the tables with the basic expressions are completed. Let us take, as an example, a table having three rows, at the front of which $k, l$ and $k+l$ are written, respectively and where $k$ and $l$ denote summands and $k+l$ there sum. While the values for $k$ and $I$ run along first two rows, their sum is calculated and written in the third row.

When children have gathered together a lot of experience of the use of letters in the situations that we have just touched upon, they are prepared well to accept easily the replacement of specific numbers by letters in the relations which have invariant form.

\section{Concluding Remarks}

When the use of letters becomes a routine practice, children and in particular students in upper classes of elementary school will be able to follow derivation of rules of algebra when data used to denote the extent of schemes are letters (without starting first with specific numbers as it is done when such derivation begins at the primary level). In our papers [7] and [8], derivation of these rules is presented in the system of natural numbers, from which they are carried over to the extended systems.

Looking closely at a number of different textbooks in mathematics for upper classes of elementary school, the following question arose: Which rules and with which reason are pointed out to be a basis for transformation of literal expressions. Scarcely have I found some of them. In any case not enough to say they constitute a complete set of rules with reason. The current research is mostly directed towards primary level and semiotic questions of symbolism. Thus the research of didactical shaping of algebraic content at the level of upper classes of elementary school has to be a matter of high interest.

Curricular innovations are successful only if the teachers are well acquainted with the new content. For that purpose, these contents have to be digested and served to them, simulating the classroom practice. Such digested contents have also to be included in didactics courses at the institutions where teachers are educated (without being afraid it will lower the academic level of such courses).

The great classical ideal, the Euclid's Elements [9], is no longer a paragon for arithmetic teaching and learning. In the Elements, arithmetic is embedded in 
geometry and the properties of proportions are basic in the operating with magnitudes (and numbers). As the phenomena whose experience produces the meaning of natural and rational numbers are discrete realities (sets at the sensory level), the Euclid's geometric model is rather a formal and narrow framework of meaning for the building of these number systems. On the other hand, the proportions do not have such a fundamental position as they had in the Elements. Multiplication as an always feasible operation in the set of natural numbers comes prior to division and the rule: $a: b=c \cdot d$ if and only if $a \times d=b \times c$, sets the interdependence between ratios (quotients) and products (when these quotients exist in the set of natural numbers). In the Elements, when proofs are carried out the word "magnitude" is used as a rhetoric variable, often illustrated as a line segment. In the contemporary mathematics, letters denote variables and serve for symbolic expression of properties of operations as well as for the formulation of statements and derivation of their proofs. This role that letters have emphasizes the importance of elaboration of algebra in school. We hope that this paper, together with the two our papers cited here, form a whole which can be helpful for further propagation of teaching and learning school algebra.

\section{Conflicts of Interest}

The author declares no conflicts of interest regarding the publication of this paper.

\section{References}

[1] Thom, R. (1974) Modèle mathématiques de la morphogenèse. Christian Bourgois, Paris.

[2] Sutherland, R., Rolano, T., Bell, A. and Lins, R. (2001) Perspectives on School Algebra. Kluwer Academic Publishers, Dordrecht.

[3] Cai, J. and Knuth, E. (Eds.) (2011) Early Algebraization, A Global Dialog from Multiple Perspectives. Springer, Berlin.

[4] Kieran, C., Pang, J.S., Schilter, D. and Ng, S.F. (2016) Early Algebra, ICME-13 Topical Surveys. Springer, Berlin.

[5] Cantor, G. (1895) BeitragezurBegrundung der transfinitenMengenlehre, Math. Annalen, 46, 481-512. https://doi.org/10.1007/BF02124929

[6] Vygotskii, A. S. (1950) Thought and Language. Izbrannyepsihologisheskieisledovaniya, Moscow. (In Russian)

[7] Marjanović, M.M. (2017) A Survey of the System $N$ of Natural Numbers Assigned to Primary Teachers. Open Access Library Journal, 4, 1-18.

[8] Marjanović, M.M. (2018) Extensions of the System $N$ of Natural Numbers Assigned to Primary Teachers. Open Access Library Journal, 5, 1-13.

[9] Euclid (2003) Euclid's Elements of Geometry. Edited and Provided with a Modern English Translation by Richard Fitzpatrick. 